\title{
Magnetic Circular Dichroism Instrumentation at LNLS
}

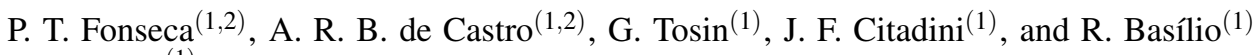 \\ (1) Laboratório Nacional de Luz Sincrotron LNLS, Campinas, SP, Brazil \\ (2) DFMC, IFGW UNICAMP, Campinas, SP, Brazil
}

Received on 2 August, 2007

\begin{abstract}
We describe the high-field $(1.0 \mathrm{~T})$ ultra high vacuum $\left(1.610^{-9} \mathrm{mbar}\right)$ controllable temperature $\left(8^{\circ} \mathrm{K}\right.$ up to $>350^{\circ} \mathrm{K}$ ) MCD setup designed and built at LNLS. We show, for nanocrystals of Hematite $\alpha-\mathrm{Fe}_{2} \mathrm{O}_{3}$, illustrative results obtained at room and at low temperature.
\end{abstract}

Keywords: MCD; Dichroism; Hematite; Nanocrystal; Spectroscopy; Synchrotron light

\section{INTRODUCTION}

Magnetic Circular Dichroism means a dependence on the magnetic field applied to the sample, of its optical absorption coefficient for circularly polarized light. The effect can be seen comparing the absorption spectra with magnetic field $\mathbf{B}$ aligned parallel and antiparallel with the photon propagation vector $\mathbf{k}[1]$.

The fundamental physical explanation of this phenomenon invokes several quantum-mechanical principles such as electronic transitions between an occupied core state and an empty valence state in the presence of a magnetic field $\mathbf{B}$, angular momenta of the electronic states and of the circularly polarized photons, conservation of energy and angular momentum and spin-orbit interaction.

The detailed theory [2-4] is rather complex, but the final result is that, from the spectra gathered with a given choice of circular polarization but opposite directions of the magnetic field $\mathbf{B}$, one can extract the separate contributions to the atomic magnetic moment, due to the intrinsic and orbital angular momenta of the electrons responsible for the light absorption process. Such quantities can be calculated directly from "first-principle" models by making hypotheses regarding the electronic quantum states. They depend critically on the chemical state and neighborhood of the atoms contributing to the absorption.

It is recalled that the magnetic (and often the transport) properties of materials containing transition metal and or rare earth atoms are related to the incomplete $3 \mathrm{~d}$ (in the transition metals) or $4 \mathrm{f}$ (in the rare earths) electronic shell. To study optical absorption involving these electrons, one needs photon energies in the range from 400 to $1500 \mathrm{eV}$, which are available only at synchrotron light sources.

From a practical point of view, MCD is a powerful method for basic research in atomic and solid state physics. For instance, metal-insulator transitions related to the $\mathrm{Ni} 3 \mathrm{~d}$ states in $\mathrm{RNiO}_{3}$ perovskites [5] have been investigated at LNLS using optical absorption. It is known that $\mathrm{Ni}$ perovskites exhibit a wealth of magnetic phase-transitions as a function of temperature [6] which could also be investigated by MCD in the same LNLS beamline, with the equipment described below. The experiment would be almost identical to [5] and perhaps allow for further elucidation of these complex materials.

The instrumentation needed for MCD measurements in most standard magnetic materials includes therefore a tun- able light source in the soft X-ray spectral range ("soft X-ray beamline" in a synchrotron facility), equipment to measure optical absorption, a device to apply a magnetic field $\mathbf{B}$ to the sample and a cooling/heating system if one wants to change the sample temperature. Since light in this spectral range is strongly absorbed by all solid materials, there can be no windows between the synchrotron source and the sample. The whole experiment has to be enclosed in the same ultra high vacuum needed for the operation of the synchrotron source.

At the Brazilian synchrotron source LNLS [7] there are two beamlines [8,9] available for such experiments, capable of delivering circularly polarized light, and a third one is under construction.

In section II we describe the MCD equipment without further discussion of the light source. In section III we show and discuss illustrative MCD spectra. In section IV we list conclusions and perspectives.

\section{DESCRIPTION OF THE MCD EQUIPMENT}

Let us consider separately the (1) probe, the (2) light absorption measurement system, the (3) magnetic system and the (4) vacuum chamber.

(1)Probe : this is a copper block supporting up to 6 samples (of size $\sim 1 \times 1 \mathrm{~cm}^{2}$ ), see Fig. 1 .

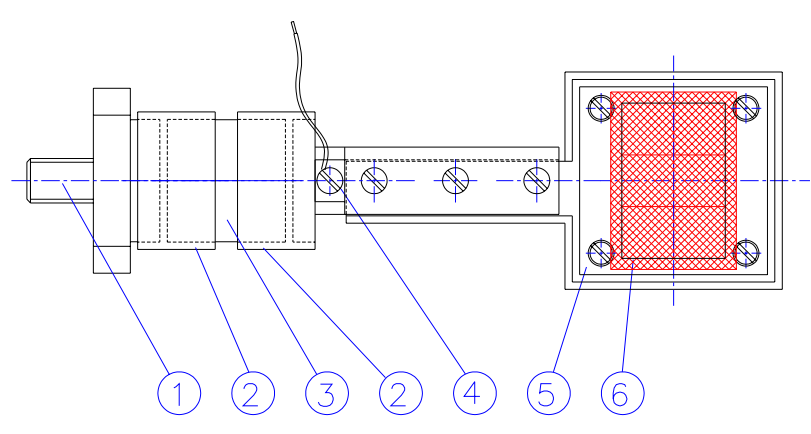

FIG. 1: Sample module: (1) Connection to the cryostat cold head; (2) Kovar thermal stress relief sleeves; (3) Alumina insulator, brazed to the sleeves; (4) Electrical connection; (5) Sample holder as such; (6) High transmission mesh, electrically insulated from the sample holder, and to which we apply a positive bias. 
The copper block is actually a cooled/heated substrate with temperature control, electrically connected to a picoammeter. The electrically insulated probe is mounted to a Helium cryostat cold finger/heater, and is exposed to soft X-ray light inside an ultra high vacuum chamber, in the presence of a magnetic field. The whole cryostat is mounted to the vacuum chamber through a mechanical feed-thru and is moved in the plane perpendicular to the light beam to select the sample one wants to expose.

Attached to the probe, $0.5 \mathrm{~mm}$ in front of the samples, there is an insulated grid (high transparency wire mesh) which is part of the detection system (see below), and which is electrically biased (about $+200 \mathrm{~V}$ ) in relation to the chamber by an external power supply.

We have three Si temperature sensors; sensor A is attached to the cold head, sensor B is attached to the probe as close as possible to the alumina insulator, sensor $\mathrm{C}$ is as close as possible to the samples. The temperature of all samples is expected to be the same because they are all very close to each other on a copper substrate with high thermal conductance.

The lowest sample temperature achievable is determined by black-body radiative heating of the cooled sample substrate and by the thermal conductivity of the alumina electrical insulator. We estimated the radiation power density (due to the $300 \mathrm{~K}$ environment) as $0.1 \mathrm{Wcm}^{-2}$ strad $^{-1}$ using the Stefan-Boltzmann law $\partial^{2} P / \partial A \partial \Omega=\sigma T^{4}$. The estimated power absorbed by each face of area $\mathrm{A}=3 \times 3 \mathrm{~cm}^{2}$ is then $2 \pi \mathrm{A}$ $\partial^{2} \mathrm{P} / \partial \mathrm{A} \partial \Omega \sim 3 \mathrm{~W}$. This estimate is consistent with the measured temperature difference $\Delta \mathrm{T}=13 \mathrm{~K}$ across the alumina insulator $(\kappa=0.074 \mathrm{Wcm} / \mathrm{K})$. At room temperature sensors A B C gave the same reading within $0.5 \mathrm{~K}$, which we take as indication of good match of the origin in their individual temperature scales, but sensor $\mathrm{C}$ has not been calibrated over the whole temperature range of interest.

There were temperature gradients in the sample probe itself. We ended up with a measured sample temperature of 45 $\mathrm{K}$ (for cold head at $8 \mathrm{~K}$ ). Also, we should note that on successive heat-cool cycles the thermal contact between the probe and its temperature sensor $\mathrm{C}$ seemed to be adversely affected. Considering the dispersion of the readings on successive heatcool cycles, we estimate the uncertainty in the absolute sample temperature (given by sensor $\mathrm{C}$ ) to be about $\pm 5 \mathrm{~K}$.

(2) Light absorption measurement: we measured the relative optical absorption indirectly, via the total electron yield, see Fig. 2. As the sample absorbs photons, it emits photoelectrons, Auger electrons and secondary electrons resulting from scattering of the former. These outgoing electrons are collected by the positively biased mesh mentioned in the previous item. The sample becomes positively charged and drains a small current (a few nA) which is measured by the picoammeter. The sample current is recorded by a computer which also selects the photon energy (wavelength) incident on the sample and has on-line access to information regarding photon beam intensity, wavelength, timing, and similar data needed for calibration and normalization of the spectra.

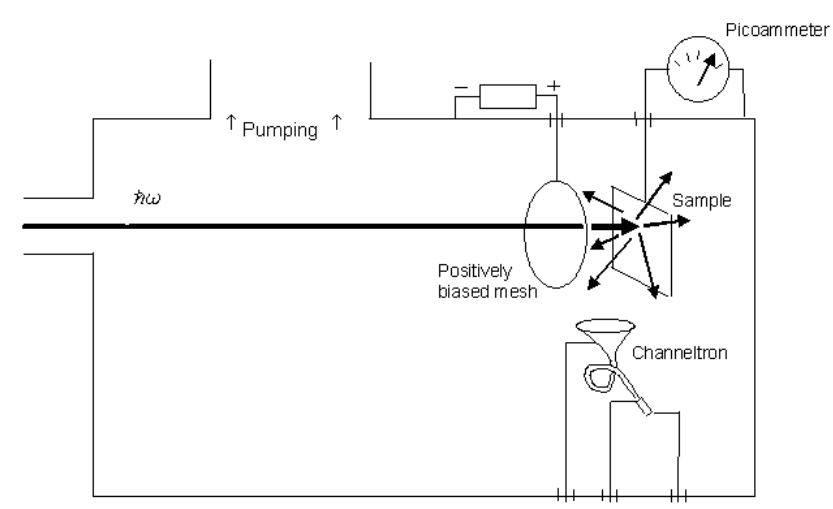

FIG. 2: Measurement of optical absorption via total electron yield.

Let us observe that many factors affect the quality of the recorded absorption spectra: spectral resolution, acquisition time, stability of the synchrotron source, value of the bias voltage applied to the electron collection grid. Furthermore, spectra taken with polarized light from a bending magnet synchrotron source (our case) pay a price in signal to noise ratio and length of acquisition time: first, one must select just a fraction of the synchrotron light beam, either above or below the plane of the orbit; second, the light intensity incident on the sample becomes very sensitive to disturbances in the stored electron beam.

(3) Magnetic system: this is a rather complex assembly made with high performance $\mathrm{NdFeB}$ permanent magnets (Vacodym $745 \mathrm{HR}$, supplied by Vacuumschmelze $\mathrm{GmbH}$, Hanau, Germany; remanence $\mathrm{B}_{r}=1.44 \mathrm{~T}$, coercivity $\mathrm{H}_{c B}=14.0$ kOe; for best UHV performance the blocks are uncoated), see Fig. 3. The magnets along the photon beam axis have a hole to let the beam thru and also for alignment purposes.

The magnetic structure is of Halbach type [10], providing maximum magnetic flux inside and minimum outside the magnet assembly. The design was made using the ESRF program Radia [11]; it was optimized for highest possible field with highest possible homogeneity at the sample positions. After assembly was completed in the magnet laboratory at LNLS, the device was characterized in great detail. We achieved a field $\mathrm{B}=1.0 \mathrm{~T}$, which is uniform to within 0.001 $T$ inside a cylindrical volume with about $1 \mathrm{~cm}$ diameter and 2 $\mathrm{mm}$ thickness, and where the sample is supposed to be placed. The magnet assembly offers two such sites, spaced by $50 \mathrm{~mm}$, where $\mathbf{B}$ points in opposite directions.

In order to choose $\mathbf{B}$ parallel or antiparallel to the photon wavevector $\mathbf{k}$, the whole magnet assembly can be moved inside the vacuum chamber around the stationary sample module, under computer or manual control, driven by a pneumatic piston.

(4) Vacuum chamber: we used a chamber with the standard LNLS design, in which the bulky magnet assembly, the cryostat and sample module, plus all the monitoring and vacuum 


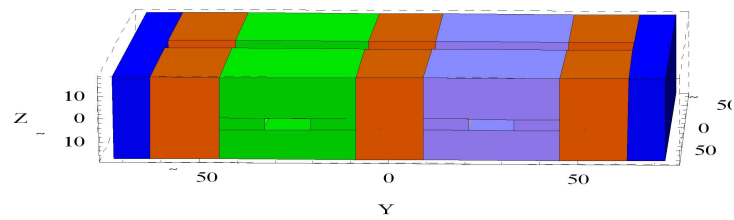

FIG. 3: The magnetic system. Please, refer to the XYZ coordinate system embedded in the figure. The positions where the field has maximum strength and homogeneity are $(0,-25,0)$ and $(0,+25,0)$, in units of $\mathrm{mm}$. In these two points, $\mathbf{B}= \pm \mathrm{B}_{0} \mathbf{x}$, with $\mathrm{B}_{0}=1$ Tesla. The rather flat sample holder can be maneuvered along the XYZ directions over some small spatial range, for fine adjustment. The whole magnetic assembly can be displaced along the $\mathrm{Y}$ axis by $50 \mathrm{~mm}$, in order to choose the direction $( \pm \mathbf{x})$ of the field applied to the sample. The focused photon beam comes along the $\mathrm{X}$ axis, goes through the rectangular holes and hits the sample. The 4 identical permanent magnet blocks around each of the two possible sample positions have their magnetic moment $\mathbf{M}$ aligned in the $( \pm \mathbf{x})$ directions; these blocks were molded with a shallow recess and joined symmetrically to make the rectangular holes. The other 6 blocks, also identical, but without recesses, have $\mathbf{M}$ along $( \pm \mathbf{y})$, in order to optimize the field in the two sample locations, and minimize the stray fields outside the structure (see Ref [10]). The two soft iron end-pieces close the magnetic circuit.

accessories fitted neatly.

The chamber was built at LNLS using the established techniques for welding and cleaning needed for ultra high vacuum parts. After bake-out at $150 \mathrm{C}$ during 24 hours, the pressure in these chambers is consistently in the low $10^{-10}$ mbar range. In our case, however, the equipment could not be heated up to such high temperature under penalty of irreversible magnetization loss. Instead of baking at high temperature, we had week long pumping down periods at $\mathrm{T}=50 \mathrm{C}$, and adopted special venting procedures whenever it was needed to break the vacuum. We were able to make our measurements at a chamber pressure in the high $10^{-10}$ mbar range. The composition of the residual gases was monitored with a dedicated RGA head mounted to one of the ports in the chamber; we detected only $\mathrm{H}$ and some $\mathrm{H}_{2} \mathrm{O}$. Water contamination at low temperature is a real concern if one wants to measure absorption near the $\mathrm{O} \mathrm{K}$ edge in water-free samples.

Pumping was provided by a large $(1000 \mathrm{l} / \mathrm{sec})$ turbomolecular pump plus a liquid Nitrogen insert, which operated as cryopump. In addition, the cryostat itself provided some nonintentional cryopumping when it was on.

\section{RESULTS AND DISCUSSION}

The samples used were aqueous solutions of colloidal $\alpha$ $\mathrm{Fe}_{2} \mathrm{O}_{3}$, prepared by R D Zysler et al in Centro Atomico Bar- iloche, Argentina, according to the procedure described in ref [12]. Several nanoparticle sizes were available. A drop of the solution was spread over the gold surface of the sample holder, and let dry at room temperature. We loaded 4 different types of nanocrystals in our sample holder. The two blank sites were used for monitoring chamber contamination.

The measurements were done at the D08-SGM beamline of the Brazilian Synchrotron Source LNLS.

For each sample, at each temperature, data gathering and treatment was as follows.

We recorded a group of spectra with fixed handedness (left or right) of the incident polarized light, inverting $\mathbf{B}$ for each successive spectrum in a group (the direction of $\mathbf{B}$ was changed by moving the magnet assembly). The rationale for such a procedure was that we needed to compute differences between acquired spectra; we hoped to cancel out slow random drifts of light beam position or intensity, by comparing spectra taken immediately one after the other.

Next, for each spectrum, we normalized the sample photocurrent to $100 \mathrm{~mA}$ stored electron beam current. After that, we removed a constant background and normalized to unit intensity of the highest peak. Finally, we got four "difference spectra" by making the difference between "adjacent" spectra with opposite $\mathbf{B}$ and took the average of the various "difference spectra"; the result is referred to as "dichroism spectrum". The "unpolarized spectrum" was obtained with unpolarized synchrotron light, or by averaging spectra taken with polarized light but opposite $\mathbf{B}$.

Figure $4 \mathrm{a}$ shows the unpolarized spectrum and Fig. $4 \mathrm{~b}$ the dichroism spectrum of sample \#422 (particles with diameter $\phi=15 \mathrm{~nm}$ ) at room temperature, in the spectral region of electronic transitions between the $\mathrm{Fe} 2 \mathrm{p}$ and $3 \mathrm{~d}$ states (Fe L edge).

A rough interpretation of the unpolarized spectrum for a single $\mathrm{Fe}$ atom is as follows: the six $2 \mathrm{p}$ states $(l=1, s=1 / 2)$, which would be degenerate in the $\mathrm{H}$ atom, are split in the $\mathrm{Fe}$ atom due to spin-orbit interaction, into two groups with total angular momentum $j=1 / 2$ and $j=3 / 2$. The ten $3 \mathrm{~d}(l=2, s=1 / 2)$ states have negligible spin-orbit interaction and are all degenerate. Transitions from $2 \mathrm{p} j=3 / 2$ into $3 \mathrm{~d}$ are roughly twice more probable than transitions from $2 \mathrm{~d} j=1 / 2$ into $3 \mathrm{~d}$, because there are four $j=3 / 2$ states as compared with only two $j=1 / 2$ states. Hence, the strong peak centered at about $708 \mathrm{eV}$ is related to initial states $j=3 / 2$, while the smaller peak at about $721 \mathrm{eV}$ is related to initial states $j=1 / 2$. The spacing between the spectral peaks is the spin-orbit interaction energy.

In the presence of the magnetic field the electronic states change. In addition, the transition probability depends not only on the number of available initial and final states, but also on the matrix element of the photon-electron interaction and on the handedness of the photons, which is why there is dichroism.

For a $\alpha-\mathrm{Fe}_{2} \mathrm{O}_{3}$ nanocrystal this simple picture has to be modified [13]. The nanocrystals are expected to have a permanent magnetic moment which depends on particle size and temperature, and which provides a local magnetic field $\mathrm{B}_{l o c}$ usually much larger than the externally applied field $\mathrm{B}_{\text {ext }}$. In a typical sample, the light beam illuminates many nanocrystals, which, in the absence of $\mathrm{B}_{e x t}$, are randomly oriented, so 


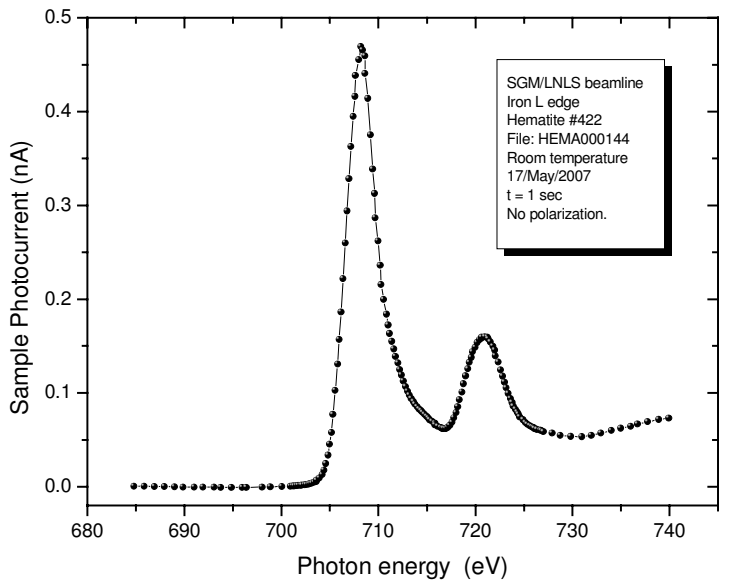

(a)

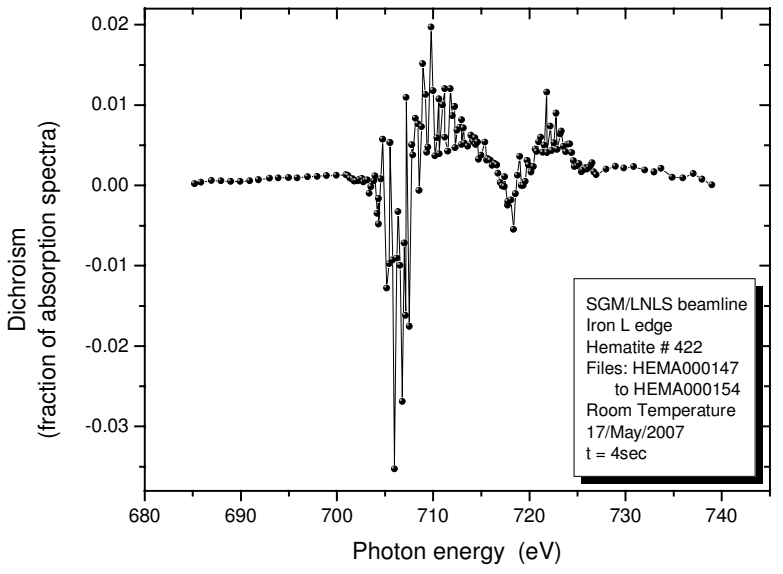

(b)

FIG. 4: Spectra of sample \#422 $\left(\alpha-\mathrm{Fe}_{2} \mathrm{O}_{3}\right)$ at room temperature, at the $\mathrm{Fe} \mathrm{L}$ absorption edge. (a) unpolarized; (b) dichroism.

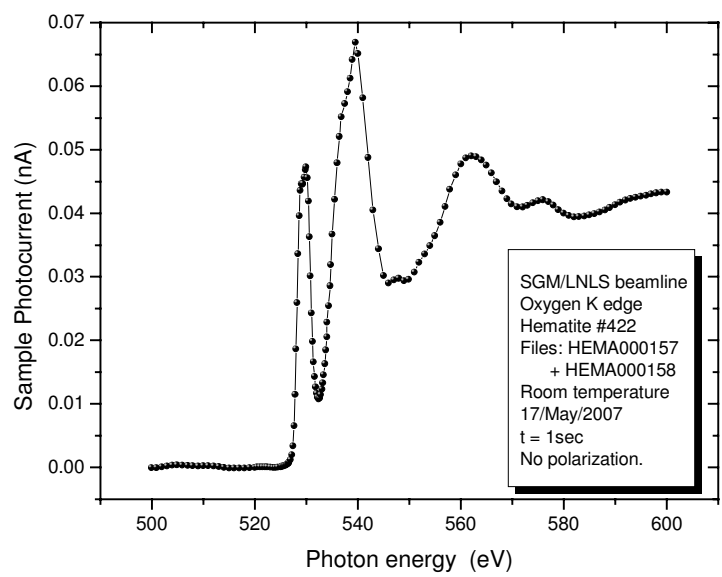

(a)

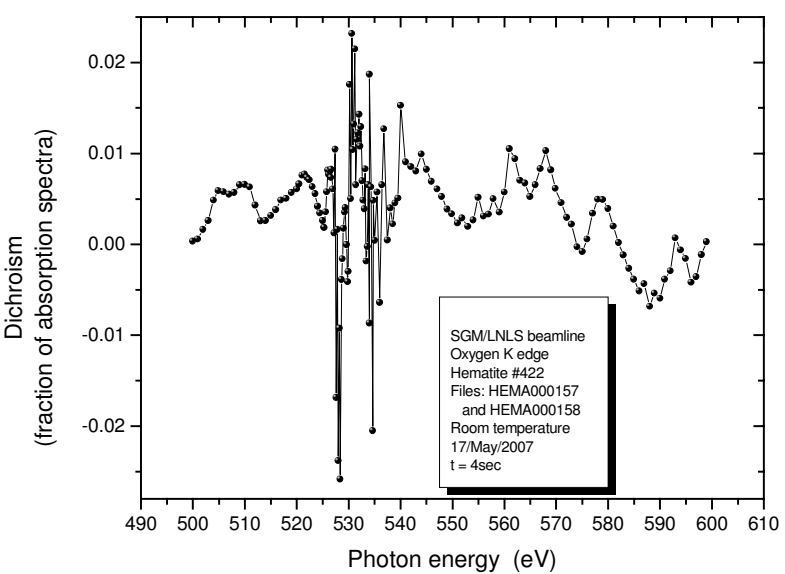

(b)

FIG. 5: Spectra of sample $\# 422\left(\alpha-\mathrm{Fe}_{2} \mathrm{O}_{3}\right)$ at room temperature, at the $\mathrm{O} \mathrm{K}$ absorption edge (a) unpolarized; (b) dichroism.

an average spectrum would always be measured. We apply the external field in order to orient the magnetic moments of all the nanocrystals parallel, and only then we see dichroic effects. The crystal structure in the nanocrystal also has strong effects on the electronic states.

Since one expects the $\mathrm{O}$ neighbors to affect the Fe electronic states and vice-versa, it is interesting also to look for dichroism at the $\mathrm{O}$ absorption edge related to transitions $1 \mathrm{~s}$ into $2 \mathrm{p}$ and $3 \mathrm{p}(\mathrm{O} \mathrm{K}$ edge $)$. In hematite the $\mathrm{O} 2 \mathrm{p} 3 \mathrm{p}$ states hybridize strongly with Fe $3 \mathrm{~d} 4 \mathrm{~s}$ [14], which results in a complicated spectral shape, from which, however, it is possible to obtain some structural information [15].

Figure 5 shows the unpolarized and dichroism spectra related to the Oxygen K edge, for sample \#422 at room temperature.

At low temperature we expect changes in the nanocrystal magnetization $[13,16]$ and in the electronic states of both $\mathrm{Fe}$ and $\mathrm{O}$, which would affect the spectra. For instance, the dichroism at the Fe L edge, Fig. 6b, is much stronger at low temperature, as expected from the discussion in [16].
Since there is no noticeable change in the spectra at the Fe L edge for the cold sample (compare Figs. 6a, 4a) we believe there is no significant water condensation, in spite of water being always present in the residual gas of the chamber. Further evidence against the possibility of significant water condensation on the sample surface at low temperature is the fact that the $\mathrm{O} \mathrm{K}$ edge spectra of the blank slot (not shown here) in the sample holder at low and room temperature are almost identical and much weaker than the spectra of sample \#422.

Figure 7 shows spectra at the $\mathrm{O} \mathrm{K}$ edge, for the cryostat temperature set to $\mathrm{T}=8 \mathrm{~K}$. The unpolarized and also the dichroism spectra are different from that shown in Fig. 5. Since there is no evidence of water condensation in the cold sample, we believe that the differences in the $\mathrm{O} \mathrm{K}$ edge spectra, at room and low temperature (Figs. 5 and 7) are a real effect associated with changes in the electronic states of the sample.

A detailed discussion of the temperature dependence of our spectra at the $\mathrm{O} \mathrm{K}$ edge will be given elsewhere. 


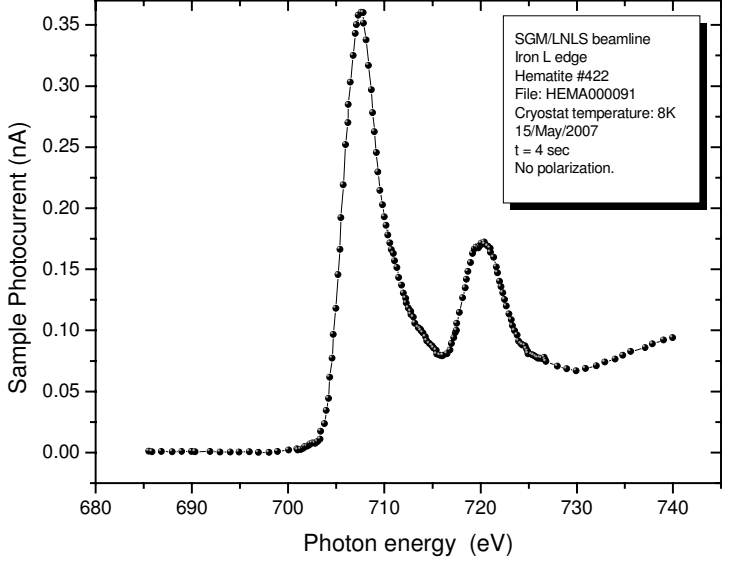

(a)

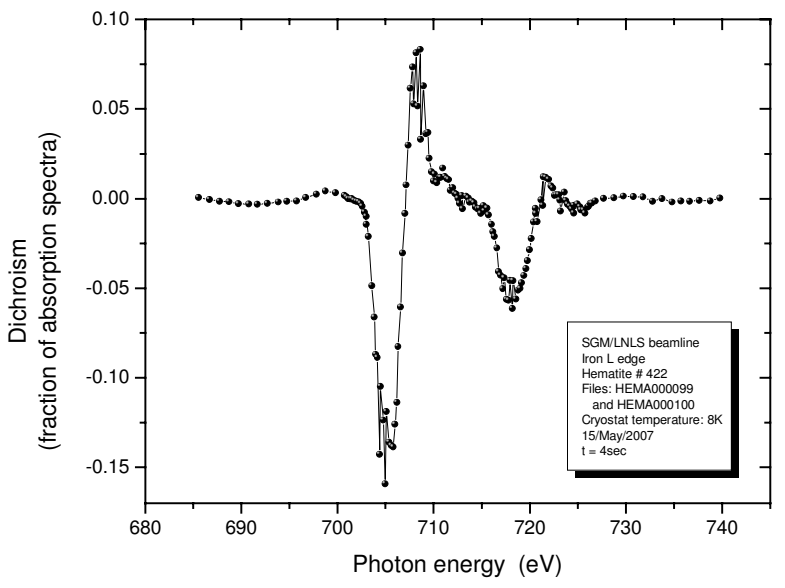

(b)

FIG. 6: Spectra of sample \#422 $\left(\alpha-\mathrm{Fe}_{2} \mathrm{O}_{3}\right)$ at cryostat head temperature $8 \mathrm{~K}$ and sample estimated temperature $45 \mathrm{~K}$, at the Fe L absorption edge. (a) unpolarized; (b) dichroism.

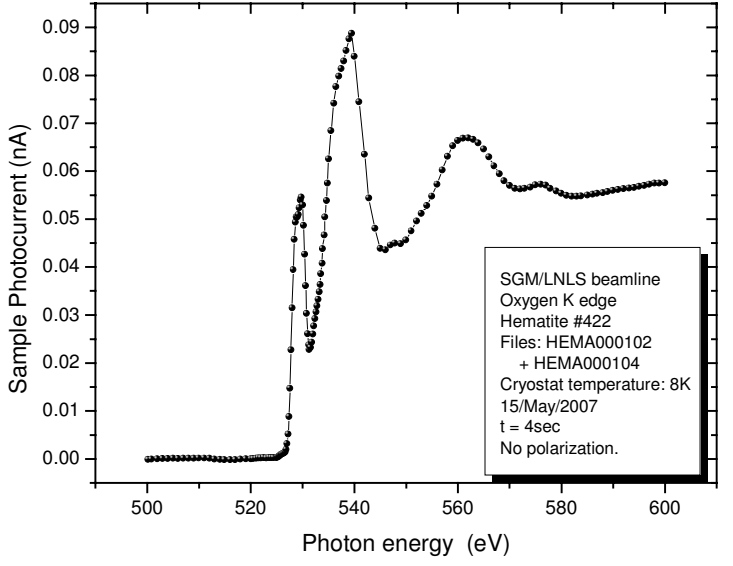

(a)

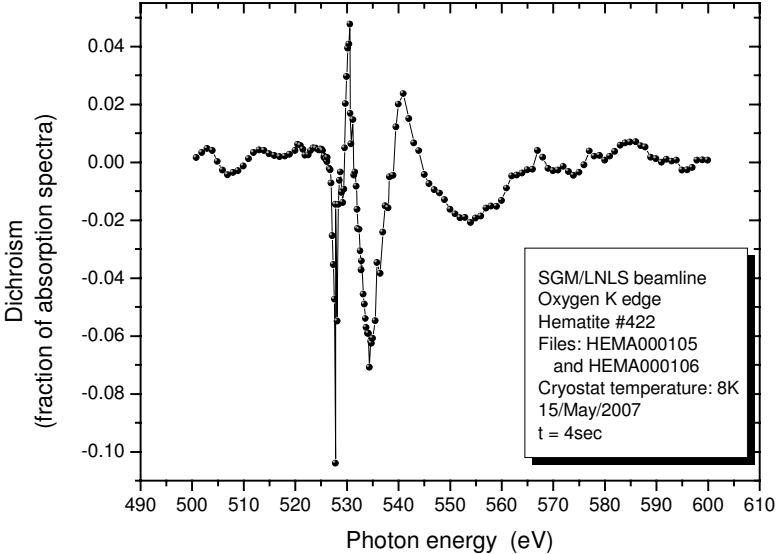

(b)

FIG. 7: Spectra of sample $\# 422\left(\alpha-\mathrm{Fe}_{2} \mathrm{O}_{3}\right)$ at cryostat head temperature $8 \mathrm{~K}$ and sample estimated temperature $45 \mathrm{~K}$, at the $\mathrm{O} \mathrm{K}$ absorption edge. (a) unpolarized; (b) dichroism

\section{CONCLUSION AND PERSPECTIVES}

We described equipment for magnetic circular dichroism measurements built at LNLS and used in the SGM (Spherical Grating Monochromator) beamline. The apparatus is compatible with ultra high vacuum $\left(10^{-10}\right.$ mbar range) and allows cooling/heating of the sample in vacuum with fine $( \pm 0.1 \mathrm{~K})$ temperature control in the range $350 \mathrm{~K}$ down to $8 \mathrm{~K}$.

The magnetic field applied to the sample is highly uniform over a large volume (cylindrical region of diameter $1 \mathrm{~cm}$ and thickness $2 \mathrm{~mm}$ ) and very strong (1 T) allowing a high degree of magnetic domain orientation even for "hard" magnetic samples.

It has so far been successfully used for measurements on thin films of hematite nanocrystals at low temperature.

Some interesting technical issues that had to be addressed are as follows: 1) Assembly of the magnetic structure, given the strong forces applied on each subsequent block, once one (or more, from a total of 14 blocks) is in place. The LNLS Magnet Group developed a special rig for handling the blocks. 2) Robust mechanical mounting of the electrically insulated probe to the cryostat cold head, allowing good thermal contact. The LNLS Materials group developed a successful brazed copper/kovar/alumina part after we tried several unfruitful schemes. 3) Extraction of the electron yield: in the presence of the strong magnetic field, a space charge cloud developed in front of the sample and there was no sample current. This space charge was removed with a biased grid installed very close to the sample, covering a solid angle of essentially $2 \pi$. 4) Limitations regarding bake-out temperature due to irreversible magnetization loss in the NdBFe blocks. We are still living uncomfortably with it. The only possible solution is to compromise on the maximum field, choosing a different material for the blocks. This would be adequate for work with nanocrystals but perhaps not with "hard" magnetic samples. At any rate, a new magnet assembly would have to 
be designed and built.

We acknowledge financial help from MCT-Nanomaterials Program (sub-program nanomagnetism) which made possible the purchase of a He cryostat, the magnet blocks and vacuum equipment.
We gratefully acknowledge the successful effort of the LNLS machine shop and LNLS Materials Lab to deliver, at very short notice, various kovar/alumina brazed precision parts redesigned during commissioning of the system.
[1] MCD can also be measured by comparing the absorption spectra at fixed $\mathbf{B}$ but with opposite handedness of the circular polarization. The two schemes are shown to be equivalent by invoking invariance under symmetry operations.

[2] J. L. Erskine and E. A. Stern, Phys. Rev. B 12, 5016 (1975).

[3] B. T. Thole, P. Carra, F. Sette, and G. van der Laan, Phys. Rev. Lett, 68, 1943 (1992).

[4] P. Carra, B. T. Thole, M. Altarelli, and X Wang, Phys. Rev. Lett. 70, 694 (1993).

[5] C. Piadmonteze, F. M. F. de Groot, H. C. N. Tolentino, A. Y. Ramos, N. E. Massa, J. A. Alonso, and M. J. Martinez-Lope Phys. Rev. B 71, 020406 (2005)

[6] C. Schinzer, Journal of alloys and compounds 288, 65 (1999).

[7] Visit the site www.lnls.br

[8] P. T. Fonseca, J. G. Pacheco, E. d'A Samogin, and A. R. B. de Castro, Rev. Sci. Instrum. 63, 1256 (1992).

[9] M. Abbate, F. C. Vicentin, V. Compagnon-Cailhol, M. C. Rocha, and H. Tolentino, J. Synchr. Rad. 6, 964 (1999).
[10] K. Halbach, Journal de Physique 44, C1-211 (1983).

[11] Visit the site www.esrf.eu/Accelerators/Groups/InsertionDevices/Software/Radia

[12] A. R. B. de Castro, R. D. Zysler, M. Vasquez-Mansilla, C. Arciprete, and M Dimitrijewits, J. Mag. Mag. Mat, 231, 287 (2001).

[13] R. A. Borzi, S. J. Stewart, G. Punte, R. C. Mercader, M. Vasquez-Mansilla, R. D. Zysler, and E. D. Cabanillas, J. Mag. Mag. Mat. 205, 234 (1999).

[14] F. M. F. de Groot, M. Grioni, J. C. Fuggle, J. Ghijsen, G. A. Sawatsky, and H. Petersen, Phys. Rev. B 40, 5715 (1989).

[15] C. R. Natoli, in "EXAFS and Near Edge Structure", vol 27 of Springer Series in Chemical Physics, pg 43, edited by A. Bianconi, L. Incoccia, and S. Stipcich, Springer, Berlin (1983).

[16] A. R. B. de Castro and R. D. Zysler, J. Mag. Mag. Mat, 25751 (2003). 